\title{
Índice de manejo de carbono e atributos químicos de Latossolo Vermelho sob diferentes sistemas de manejo
}

\author{
Jolimar Antonio Schiavo(1), Jean Sérgio Rosset( ${ }^{(1)}$, Marcos Gervasio Pereira(2) e Júlio Cesar Salton ${ }^{(3)}$
}

\begin{abstract}
(1)Universidade Estadual de Mato grosso do Sul, Rodovia Aquidauana, Km 12, CEP 79200-000 Aquidauana, MS. E-mail: schiavo@uems.br (2)Universidade Federal Rural do Rio de Janeiro, Departamento de Solos, BR 465, Km 7, CEP 23890-000 Seropédica, RJ. E-mail: gervasio@ufrrj.br

(3)Embrapa Agropecuária Oeste, Rodovia BR 163, Km 253, CEP 79812-240 Dourados, MS. E-mail: salton@cpao.embrapa.br
\end{abstract}

\begin{abstract}
Resumo - O objetivo deste trabalho foi avaliar mudanças nos atributos químicos, no estoque de carbono e no índice de manejo de C de um Latossolo Vermelho argiloso, sob diferentes sistemas de manejo. Avaliou-se o solo sob sistemas de manejo com diferentes históricos de uso, sob as seguintes coberturas: Zea mays, Urochloa decumbens e Panicum maximum cv. Tanzania. Como testemunha, avaliou-se área sob vegetação de cerrado. Foram coletadas amostras compostas das camadas de solo de 0-5, 5-10 e 10-20 cm. Os teores de fósforo diminuíram com a profundidade, e os maiores valores foram observados na área com $P$. maximum. $\mathrm{Na}$ área com milho, observaram-se maiores valores de $\mathrm{K}^{+}, \mathrm{Ca}^{++}, \mathrm{Mg}^{++}, \mathrm{CTC}$, e de soma e saturação por bases. $\mathrm{O}$ teor de $\mathrm{C}$ orgânico total do solo (COT) foi maior sob cerrado e menor sob cobertura com P. maximum, à profundidade de 5-10 cm. Os maiores estoques de COT, carbono nas frações particuladas (C-MOP) e nas frações associadas à fase mineral do solo ocorreram na área com $U$. decumbens, o que promoveu maiores índices de labilidade e de manejo de $\mathrm{C}$, similares aos da vegetação de cerrado. Em todas as coberturas, a labilidade da matéria orgânica do solo diminuiu com o aumento da profundidade. A fração C-MOP apresenta maior sensibilidade às alterações de manejo e relaciona-se ao COT.
\end{abstract}

Termos para indexação: Cerrado, índice de labilidade, integração lavoura-pecuária, manejo de solo, matéria orgânica do solo, sustentabilidade do solo.

\section{Carbon management index and chemical attributes of an Oxisol under different management systems}

\begin{abstract}
The objective of this work was to evaluate changes in the soil chemical attributes, carbon stock and in the $\mathrm{C}$ management index of a clayed Oxisol under different management systems. The soil under management systems with different historical usage was evaluated under the following covers: Zea mays, Urochloa decumbens, Panicum maximum cv. Tanzania. A cerrado area was used as check. Four composite samples were collected at $0-5,5-10$ and $10-20 \mathrm{~cm}$ soil depths. The phosphorus contents decreased with depth, and the higher values were observed in the area with $P$. maximum. In the area with $Z$. mays, higher values for $\mathrm{K}^{+}, \mathrm{Ca}^{++}, \mathrm{Mg}^{++}, \mathrm{CEC}$, and base sum and saturation were observed. The total organic $\mathrm{C}$ content (TOC) was higher under cerrado vegetation cover, and smaller under P. maximum at 5-10 cm soil depth. The higher stocks of TOC, and of carbon in particulate fractions (C-POM) and in fractions associate to the soil mineral phase occurred in the area with $U$. decumbens, which promoted higher values for lability and $\mathrm{C}$ management indexes, similar to those for cerrado vegetation. In all coverings, the organic soil matter lability decreased with depth. The C-POM fraction had higher sensibility to variation in management practices, and is related to TOC.
\end{abstract}

Index terms: Cerrado, lability index, integrated crop-livestock, soil management, soil organic matter, soil sustainability.

\section{Introdução}

O revolvimento contínuo e intenso do solo, com a adoção de sistemas agrícolas baseados em monocultura ou em sucessões contínuas de culturas, tem resultado na diminuição da qualidade física, química e biológica dos solos (Costa et al., 2003). O sistema de plantio direto surgiu como alternativa ao manejo convencional com preparo do solo e atingiu grande expansão na área plantada a partir do início da década de 1990 (Anghinoni, 2007).

Sistemas mistos, que integram a exploração de lavoura e pecuária (ILP), têm sido propostos pelas vantagens que apresentam em relação a sistemas isolados de agricultura ou pecuária. O sistema ILP temse tornado uma alternativa eficiente de uso da terra, pois promove a recuperação e a renovação das pastagens,

Pesq. agropec. bras., Brasília, v.46, n.10, p.1332-1338, out. 2011 
permite o uso racional dos insumos, diversifica a produção e mantém a produtividade agrícola de grãos (Spera et al., 2006; Balbinot Junior et al., 2009). O sistema ILP pode aumentar as concentrações de carbono (C) orgânico no solo ao longo do tempo, em razão do crescimento contínuo de plantas na área, da rotação de culturas, do incremento na matéria vegetal produzida por tempo, e da maior ciclagem de nutrientes (Tracy \& Zhang, 2008).

O teor de matéria orgânica do solo (MOS) é um indicador de sua qualidade (Conceição et al., 2005), já que influencia diretamente atributos físicos, químicos e biológicos do solo. Porém, a simples quantificação dos estoques de $\mathrm{C}$ total ou de suas frações não fornece valores extrapoláveis para diferentes situações de manejo, locais, climas e solos (Nicoloso et al., 2008). Neste aspecto, reservatórios lábeis ou complexados, em diferentes graus de interação com a matriz mineral do solo (MOS estável), podem ter implicações na retenção de $\mathrm{C}$ atmosférico, bem como nas propriedades físicas, químicas e biológicas dos solos. A fração lábil da MOS pode ocorrer livremente e seu mecanismo de proteção é intrínseco à recalcitrância de sua composição química ou está relacionado à oclusão no interior dos agregados, o que limita o acesso a microrganismos e suas enzimas (Christensen, 2001). A MOS estável é constituída, geralmente, por materiais mais humificados, estabilizados pela ligação direta entre as frações orgânicas e minerais (Golchin et al., 1994).

Para o fracionamento do $\mathrm{C}$ nos diferentes compartimentos, no solo, têm sido utilizadas técnicas que podem ser granulométricas (Cambardella \& Elliott, 1992; Feller \& Beare, 1997; Franzluebbers \& Arshad, 1997; Wander \& Bidart, 1998; Needelman et al., 1999), densimétricas (Golchin et al., 1994), ou uma combinação de ambas (Six et al., 1998). Pelo fracionamento granulométrico, são separados por peneiramento via úmida o $\mathrm{C}$ orgânico particulado (COP), na fração lábil maior que $53 \mu \mathrm{m}$, e o carbono orgânico associado à fração mineral $(\mathrm{COM})$, menor que $53 \mu \mathrm{m}$. De acordo com Conceição et al. (2005), a fração COP é a mais sujeita a mudanças resultantes do manejo do solo e, portanto, pode ser utilizada como indicador mais sensível da qualidade do solo.

A partir de dados do fracionamento granulométrico, é possível obter-se o índice de manejo de C (IMC), proposto por Blair et al. (1995), que relaciona o estoque de $\mathrm{C}$ do solo e sua labilidade, calculados com base num sistema de referência (vegetação em estado natural ou pastagem). O IMC parece ser uma ferramenta útil para subsidiar informações acerca dos melhores sistemas de manejo de solos e culturas, pois integra, numa mesma medida, as variações ocorridas nas diferentes frações da MOS (Nicoloso et al., 2008). Trabalhos que envolvem labilidade e IMC na região do Cerrado, sob sistema de ILP com rotação de culturas produtoras de grãos e pastagens, em áreas de longa duração, são bastante escassos.

O objetivo deste trabalho foi avaliar mudanças nos atributos químicos, no estoque de carbono e no índice de manejo de $\mathrm{C}$ de um Latossolo Vermelho, sob diferentes sistemas de manejo.

\section{Material e Métodos}

O experimento foi realizado na Fazenda Taquaruçu, no Município de Maracaju, MS, a 2137'55"S, $55^{\circ} 08^{\prime} 55^{\prime \prime} \mathrm{W}$, em Latossolo Vermelho, com textura argilosa (Santos et al., 2006). O manejo utilizado nas áreas amostradas foi o do plantio direto com integração lavoura-pecuária, com diferentes históricos de uso e manejo. À época da amostragem, em novembro de 2007, as áreas estavam cultivadas com milho (Zea mays L.), braquiária [Urochloa decumbens (Stapf) R.D.Webster], capim-tanzânia (Panicum maximum cv. Tanzânia) e, como testemunha, utilizou-se uma área de cerrado. A área cultivada com milho caracterizava-se pelo cultivo consecutivo havia três anos, com soja no verão e milho na safrinha, tendo sido anteriormente cultivada com $U$. decumbens, por cinco anos. As áreas com braquiária e campim-tanzânia estavam ocupadas com essas coberturas havia sete anos e tinham sido anteriormente ocupadas com soja, em plantio direto, pelo período de 25 anos.

De cada área, foram coletadas quatro amostras compostas, formadas de dez amostras simples, em uma área de $400 \mathrm{~m}^{2}$, das profundidades de 0-5, 5-10 e 10-20 cm. Após a coleta, as amostras foram secas e peneiradas $(2 \mathrm{~mm})$ e constituíram a terra fina seca ao ar (TFSA), tendo sido caracterizadas química e fisicamente de acordo com Claessen (1997).

$\mathrm{O}$ pH foi determinado em água (relação solo: solução 1:2,5); o $\mathrm{Ca}^{2+}$ e $\mathrm{Mg}^{2+}$ trocáveis foram extraídos com $\mathrm{KCl} 1 \mathrm{~mol} \mathrm{~L}^{-1}$ e determinados por complexiometria; o $\mathrm{Na}^{+}$e $\mathrm{K}^{+}$trocáveis foram extraídos por solução Mehlich-1 e determinados por fotometria de chama; $\mathrm{H}^{+}+\mathrm{Al}^{3+}$ foram extraídos com 
solução de acetato de cálcio a $0,5 \mathrm{~mol} \mathrm{~L}^{-1}$ tamponada a pH 7,0, e determinados por titulação; o P disponível foi extraído com solução de Mehlich-1 e determinado por colorimetria; e o C orgânico total foi determinado por oxidação com dicromato de potássio em meio sulfúrico (Claessen, 1997).

Na TFSA, a composição granulométrica foi avaliada pelo método da pipeta, tendo-se utilizado a dispersão com $\mathrm{NaOH}$ a $1 \mathrm{~mol} \mathrm{~L}^{-1}$ (Claessen, 1997). Também, de cada profundidade, foram coletadas, com auxílio de anel volumétrico de $73,5 \mathrm{~cm}^{3}$, amostras indeformadas para determinação da densidade do solo conforme Claessen (1997).

O fracionamento granulométrico da MOS foi determinado segundo Cambardella \& Elliott (1992), tendo-se obtido as frações: matéria orgânica particulada $(\mathrm{MOP},>53 \mu \mathrm{m})$ e a matéria orgânica associada a minerais (MOM, $<53 \mu \mathrm{m}$ ). A partir dos dados de COT e de densidade do solo, foi calculado o estoque de COT de cada camada e das frações granulométricas conforme Freixo et al. (2002).

A partir dos resultados do estoque de C, calculou-se o IMC, conforme Blair et al. (1995). Este índice referese à medida relativa das alterações provocadas pelo manejo do solo, em comparação com uma configuração de solo original ou ideal. Como condição original, o solo do fragmento de cerrado foi utilizado como referência (IMC $=100)$.

Para a obtenção do IMC, necessita-se do índice de estoque de C (IEC), calculado a partir da relação entre o COT de cada área e o COT da área de cerrado, utilizada como referência. A labilidade (L) da MOS foi determinada pela relação entre C-MOP e C-MOM, e o índice de labilidade (IL) foi calculado pela relação entre a labilidade de cada área e a labilidade da área de referência. O IMC de cada área foi obtido pelo IEC multiplicado pelo IL e por 100 .

Pressupondo-se o estudo como observacional, sem delineamento experimental, a comparação dos dados foi feita com o teste não paramétrico de Dunn, a $5 \%$ de probabilidade, cuja análise estatística foi realizada com auxílio do SAS. Primeiramente, os dados foram submetidos ao teste de normalidade de Shapiro-Wilk, tendo-se procedido em seguida à análise de variância pelo método de Kruskal-Wallis, em que a cobertura do solo foi a fonte de variação; e, ao final, as médias foram comparadas pelo teste de Dunn, a $5 \%$ de probabilidade.

\section{Resultados e Discussão}

A análise granulométrica mostrou que todas as áreas apresentavam textura argilosa, com teores de argila entre 430 e $540 \mathrm{~g} \mathrm{~kg}^{-1}$ (Tabela 1). Quanto à densidade do solo, as maiores variações ocorreram na camada superficial, com valores entre 0,86 e $1,60 \mathrm{Mg} \mathrm{m}^{-3}$ nas áreas de vegetação de cerrado e capim-tanzânia, respectivamente.

Os atributos químicos do solo apresentaram alterações, em razão da rotação de culturas, no sistema de integração lavoura-pecuária (Tabela 2). Nas três profundidades estudadas, os menores valores de $\mathrm{pH}$ foram observados na área de pastagem de capim-tanzânia, seguidos dos de braquiária. Os teores de $\mathrm{P}$ diminuíram conforme a profundidade, e os maiores valores ocorreram na área de pastagem de capim-tanzânia, com 10,05 e 4,69 mg kg-1 nas profundidades de $0-5$ e $5-10 \mathrm{~cm}$, respectivamente. De acordo com Schlindwein \& Gianello (2004) e Anghinoni (2007), em sistemas com manutenção da palhada, em que não é feito o revolvimento do solo, é comum a formação de gradientes de matéria orgânica e dos atributos químicos do solo, principalmente de nutrientes pouco móveis como o fósforo.

Tabela 1. Granulometria, classe textural e densidade do solo de Cerrado com diferentes coberturas vegetais, manejado sob diferentes históricos de uso e manejo ${ }^{(1)}$.

\begin{tabular}{lccccc}
\hline Cobertura do solo & $\begin{array}{c}\text { Argila } \\
\text {------- }\left(\mathrm{g} \mathrm{kg}^{-1}\right)\end{array}$ & $\begin{array}{c}\text { Silte----- } \\
\text { Areia }\end{array}$ & Textura & $\begin{array}{c}\text { Densidade do solo } \\
\left(\mathrm{Mg} \mathrm{m}^{-3}\right)\end{array}$ \\
\hline Milho & 540 & 297 & 163 & Argilosa & $1,50 \mathrm{ab}$ \\
Braquiária & 430 & 351 & 219 & Argilosa & $1,32 \mathrm{~b}$ \\
Capim-tanzânia & 447 & 295 & 258 & Argilosa & $1,60 \mathrm{a}$ \\
Cerrado & 449 & 355 & 196 & Argilosa & $0,86 \mathrm{c}$ \\
\hline & & & $5-10 \mathrm{~cm}$ & \\
Milho & 495 & 307 & 198 & Argilosa & $1,52 \mathrm{a}$ \\
Braquiária & 488 & 341 & 171 & Argilosa & $1,54 \mathrm{a}$ \\
Capim-tanzânia & 446 & 294 & 260 & Argilosa & $1,52 \mathrm{a}$ \\
Cerrado & 442 & 369 & 189 & Argilosa & $1,12 \mathrm{~b}$ \\
\hline & & & & $10-20 \mathrm{~cm}$ & \\
Milho & 505 & 316 & 179 & Argilosa & $1,56 \mathrm{a}$ \\
Braquiária & 531 & 325 & 143 & Argilosa & $1,44 \mathrm{a}$ \\
Capim-tanzânia & 453 & 300 & 247 & Argilosa & $1,50 \mathrm{a}$ \\
Cerrado & 475 & 353 & 172 & Argilosa & $0,96 \mathrm{~b}$ \\
\hline CV (\%) & - & - & - & - & 9,63 \\
\hline
\end{tabular}

${ }^{(1)}$ Médias seguidas de letras iguais, nas colunas, em cada profundidade, não diferem entre si pelo teste de Dunn, a 5\% de probabilidade. 
Os valores de $\mathrm{H}^{+}+\mathrm{Al}^{+3}$ foram elevados e variavam de $5,40 \mathrm{cmol}_{\mathrm{c}} \mathrm{kg}^{-1}(0-5 \mathrm{~cm})$, na área cultivada com milho, a $9,73 \mathrm{cmol}_{\mathrm{c}} \mathrm{kg}^{-1}(5-10 \mathrm{~cm})$ na área de cerrado. Na área cultivada com milho, observaram-se os maiores valores de $\mathrm{K}^{+}$, com $3,08,1,28$ e $0,52 \mathrm{cmol}_{\mathrm{c}} \mathrm{kg}^{-1}$, nas profundidades de $0-5,5-10$ e 10-20 cm, respectivamente. Os teores

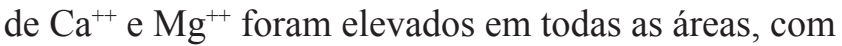
destaque para a área cultivada com milho, onde os valores de $\mathrm{Ca}^{++}$variaram de 8,92 a 9,52 $\mathrm{cmol}_{\mathrm{c}} \mathrm{kg}^{-1}$, e os de $\mathrm{Mg}^{++}$de 3,20 a 6,35 $\mathrm{cmol}_{\mathrm{c}} \mathrm{kg}^{-1}$. Destacam-se também os elevados teores desses elementos, encontrados na área sob vegetação natural de cerrado, provavelmente em razão do material de origem do solo ser formado por calcário. Proporcionalmente aos elevados teores de cátions trocáveis, constataram-se elevados valores de $\mathrm{T}\left(13,7\right.$ a $\left.35,18 \mathrm{cmol}_{\mathrm{c}} \mathrm{kg}^{-1}\right)$ e de soma de bases $(7,98$ a 26,39 $\mathrm{cmol}_{\mathrm{c}} \mathrm{kg}^{-1}$ ), o que contribuiu para valores de saturação por bases acima de $50 \%$ em todas as coberturas estudadas. Das áreas cultivadas, a de milho, principalmente nas profundidades de $0-5$ e $5-10 \mathrm{~cm}$, apresentou maiores valores de CTC e de soma e saturação por bases, em consequência das aplicações de calcário e gesso ao solo, na recente mudança de uso de pastagens para lavoura (três anos).

Nas três profundidades, o solo sob a vegetação de cerrado apresentou os maiores teores de C orgânico total (COT) (Tabela 2). Nas três áreas cultivadas, não houve diferença entre os teores de COT nas profundidades de 0-5 e 10-20 cm. Na profundidade de $5-10 \mathrm{~cm}$, as áreas com braquiária e milho apresentaram os maiores teores de COT. Houve estreita relação do COT com a CTC e a soma de bases (Figura 1).

O estoque de COT, C-MOP e C-MOM apresentou alterações em consequência das coberturas e dos sistemas de rotação de culturas utilizados no sistema de integração lavoura-pecuária avaliado (Tabela 3). Ressalta-se, no entanto, que a comparação do estoque de carbono de amostragens apenas da camada arável pode levar à subestimação dos estoques de carbono do solo, após a adoção de sistemas mais conservacionistas, como plantio direto e integração lavoura-pecuária (Boddey et al., 2008).

Na profundidade de $0-5 \mathrm{~cm}$, o maior estoque de COT ocorreu na área de pastagem com braquiária $\left(20,23 \mathrm{Mg} \mathrm{ha}^{-1}\right)$, superior inclusive à área de vegetação natural de cerrado $\left(15,0 \mathrm{Mg} \mathrm{ha}^{-1}\right)$. Na profundidade de 5-10 cm, na área com capim-tanzânia, ocorreu o menor estoque de $\operatorname{COT}\left(8,88 \mathrm{Mg} \mathrm{ha}^{-1}\right)$. Entre as demais coberturas do solo, não houve diferença significativa.

Na profundidade de $0-5 \mathrm{~cm}$, não houve diferença no estoque de C-MOP em consequência dos tratamentos (Tabela 3). Porém, nas profundidades de 5-10 e 10-20 cm, o maior estoque de C-MOP,

Tabela 2. Atributos químicos de solo de cerrado, com diferentes coberturas vegetais, manejado sob diferentes históricos de uso e manejo ${ }^{(1)}$.

\begin{tabular}{|c|c|c|c|c|c|c|c|c|c|c|c|}
\hline Cobertura do solo & $\begin{array}{c}\mathrm{pH} \\
\text { em água }\end{array}$ & $\begin{array}{c}\mathrm{P} \\
\left(\mathrm{mg} \mathrm{kg}^{-1}\right)\end{array}$ & $\mathrm{K}^{+}$ & $\mathrm{Ca}^{++}$ & $\mathrm{Mg}^{++}$ & $\begin{array}{l}\mathrm{Na}^{+} \\
\left.\mathrm{ol}_{\mathrm{c}} \mathrm{kg}^{-1}\right) \\
\end{array}$ & $\mathrm{H}^{+}+\mathrm{Al}^{+3}$ & CTC & SB & $\begin{array}{c}\mathrm{V} \\
(\%) \\
\end{array}$ & $\begin{array}{c}\text { COT } \\
\left(\mathrm{g} \mathrm{kg}^{-1}\right) \\
\end{array}$ \\
\hline & \multicolumn{11}{|c|}{$0-5 \mathrm{~cm}$} \\
\hline Milho & $6,19 \mathrm{a}$ & $2,85 b$ & $3,08 \mathrm{a}$ & $9,52 \mathrm{~b}$ & $6,35 \mathrm{a}$ & $2,56 \mathrm{a}$ & $5,40 \mathrm{~b}$ & $26,92 \mathrm{ab}$ & $21,51 \mathrm{a}$ & $79,90 \mathrm{a}$ & $20,38 b$ \\
\hline Braquiária & $6,00 \mathrm{a}$ & $1,42 b$ & $0,85 b$ & $5,05 \mathrm{c}$ & $4,95 \mathrm{a}$ & $0,81 \mathrm{a}$ & $6,68 \mathrm{ab}$ & $18,34 \mathrm{bc}$ & $11,66 b$ & $63,52 \mathrm{bc}$ & $25,58 \mathrm{~b}$ \\
\hline Capim-tanzânia & $5,30 \mathrm{~b}$ & $10,05 \mathrm{a}$ & $0,93 b$ & $3,80 \mathrm{c}$ & $3,20 \mathrm{a}$ & $0,73 \mathrm{a}$ & $7,63 \mathrm{ab}$ & $16,30 \mathrm{c}$ & $8,67 \mathrm{~b}$ & $52,66 c$ & $20,51 b$ \\
\hline \multirow[t]{2}{*}{ Cerrado } & $6,29 \mathrm{a}$ & $6,53 \mathrm{ab}$ & $2,40 \mathrm{ab}$ & $15,20 \mathrm{a}$ & $6,77 \mathrm{a}$ & $2,01 \mathrm{a}$ & $8,78 \mathrm{a}$ & $35,18 \mathrm{a}$ & $26,39 a$ & $74,43 \mathrm{ab}$ & $36,26 \mathrm{a}$ \\
\hline & \multicolumn{11}{|c|}{$5-10 \mathrm{~cm}$} \\
\hline Milho & $6,08 \mathrm{ab}$ & $2,61 b$ & $1,28 \mathrm{a}$ & $8,92 \mathrm{~b}$ & $5,2 \mathrm{a}$ & $1,38 \mathrm{a}$ & $6,23 b$ & $23,02 b$ & $16,79 a$ & $72,80 \mathrm{a}$ & $20,15 \mathrm{ab}$ \\
\hline Braquiária & $5,91 \mathrm{~b}$ & $1,13 b$ & $0,82 \mathrm{a}$ & $5,35 \mathrm{c}$ & $3,57 \mathrm{a}$ & $0,78 \mathrm{a}$ & $7,55 b$ & $18,07 \mathrm{c}$ & $10,53 b$ & $57,81 b c$ & $23,15 \mathrm{ab}$ \\
\hline Capim-tanzânia & $5,48 \mathrm{c}$ & $4,69 \mathrm{a}$ & $0,33 \mathrm{a}$ & $4,02 \mathrm{c}$ & $3,47 \mathrm{a}$ & $0,87 \mathrm{a}$ & $6,35 b$ & $14,42 \mathrm{c}$ & $8,06 \mathrm{~b}$ & $56,01 \mathrm{c}$ & $11,89 \mathrm{~b}$ \\
\hline \multirow[t]{2}{*}{ Cerrado } & $6,41 \mathrm{a}$ & $2,32 b$ & $0,99 \mathrm{a}$ & $14,5 \mathrm{a}$ & $4,37 \mathrm{a}$ & $0,23 \mathrm{a}$ & $9,73 \mathrm{a}$ & $30,47 \mathrm{a}$ & $20,74 \mathrm{a}$ & $68,02 \mathrm{ab}$ & $30,01 \mathrm{a}$ \\
\hline & \multicolumn{11}{|c|}{$10-20 \mathrm{~cm}$} \\
\hline Milho & $6,06 \mathrm{~b}$ & $1,19 \mathrm{a}$ & $0,52 \mathrm{a}$ & $9,12 \mathrm{~b}$ & $3,20 \mathrm{a}$ & $0,25 \mathrm{a}$ & $5,73 a$ & $18,83 \mathrm{~b}$ & $13,09 \mathrm{~b}$ & $69,51 \mathrm{ab}$ & $17,57 \mathrm{~b}$ \\
\hline Braquiária & $5,81 \mathrm{bc}$ & $1,19 \mathrm{a}$ & $0,42 \mathrm{a}$ & $4,75 \mathrm{c}$ & $3,35 \mathrm{a}$ & $0,24 \mathrm{a}$ & $8,74 a$ & $17,51 b$ & $8,76 b$ & $50,69 \mathrm{c}$ & $15,49 b$ \\
\hline Capim-tanzânia & $5,74 \mathrm{c}$ & $1,90 \mathrm{a}$ & $0,19 \mathrm{a}$ & $4,17 \mathrm{c}$ & $3,37 \mathrm{a}$ & $0,23 \mathrm{a}$ & $5,69 \mathrm{a}$ & $13,67 b$ & $7,98 \mathrm{~b}$ & $58,06 \mathrm{bc}$ & $11,69 b$ \\
\hline Cerrado & $6,19 \mathrm{a}$ & $2,32 \mathrm{a}$ & $1,31 \mathrm{a}$ & $11,00 \mathrm{a}$ & $7,12 \mathrm{a}$ & $1,48 \mathrm{a}$ & $6,64 \mathrm{a}$ & $27,55 \mathrm{a}$ & $20,91 \mathrm{a}$ & $75,76 \mathrm{a}$ & $28,61 \mathrm{a}$ \\
\hline$\overline{\mathrm{CV}(\%)}$ & 2,88 & 58,13 & 74,08 & 15,47 & 52,71 & 94,53 & 18,00 & 14,17 & 19,10 & 8,00 & 24,35 \\
\hline
\end{tabular}

${ }^{(1)}$ Médias seguidas de letras iguais, nas colunas, em cada profundidade, não diferem entre si pelo teste de Dunn, a $5 \%$ de probabilidade. SB, soma de bases $\left(\mathrm{Ca}^{2+}+\mathrm{Mg}^{2+}+\mathrm{K}^{+}+\mathrm{Na}^{+}\right) ; \mathrm{V}$, saturação por bases. 
depois da vegetação de cerrado, ocorreu na área com braquiária. Quanto ao estoque de C-MOM, na camada superficial de $0-5 \mathrm{~cm}$, os maiores valores ocorreram nas áreas com milho e braquiária; ao passo que às profundidades de 5-10 e 10-20 cm, destacou-se a área com capim-tanzânia, com o menor estoque de C-MOM.

Os estoques de C-MOP, em relação ao COT, variaram de 9,53 a $21,53 \%, 4,38$ a $21,82 \%$, e 3,97 a $18,63 \%$, às profundidades de $0-5,5-10$ e $10-20 \mathrm{~cm}$, respectivamente (Tabela 3). A menor resposta do estoque de C-MOP, na camada superficial, às variações de cobertura do solo pode ter ocorrido em virtude do aporte de resíduos, que foi mantido continuamente em todos os sistemas de manejo, o que corrobora os resultados encontrados por Bayer et al. (2004), em trabalho com Latossolo Vermelho, em área de cerrado manejado sob plantio direto. No entanto, nas camadas subsuperficiais, o estoque de C-MOP foi mais afetado pelos diferentes manejos adotados, e os maiores valores foram observados na área de braquiária. Esses resultados mostram que o estoque de C-MOP constitui um indicador mais sensível aos efeitos dos sistemas de manejo do solo (Chan, 1997; Needelman et al., 1999; Bayer et al., 2001, 2002, 2004).

A maior parte do estoque de COT estava ligada aos minerais do solo (C-MOM) com valores entre 78,2 e 96\% (Tabela 3). Essa fração foi pouco sensível aos

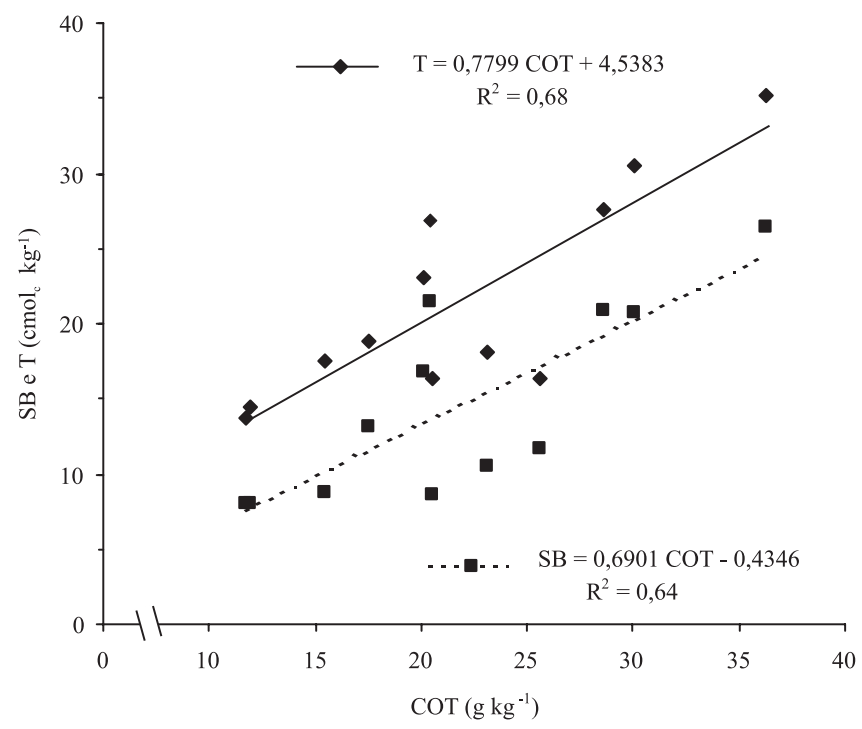

Figura 1. Relação do carbono orgânico total (COT) com o valor de CTC (T), e soma de bases (SB) de solo de cerrado, com diferentes coberturas vegetais, manejado sob diferentes históricos de uso e manejo. manejos avaliados, exceto na área com capim-tanzânia, onde se verificaram os menores valores. De maneira geral, alterações no manejo de solos, por curto período de incorporação de resíduos, resultaram em pequenas alterações nos teores de matéria orgânica associada aos minerais do solo. Esse padrão pode ser atribuído ao avançado estágio de humificação da matéria orgânica, que se torna altamente estável (estabilidade química). Outra explicação para os elevados valores desta fração seria sua proteção física pela localização no interior dos microagregados menores que $53 \mu \mathrm{m}$ (Bayer, 1996).

Os maiores IEC, na camada superficial de 0-5 cm, foram observados na área com braquiária, e os valores nas demais áreas foram semelhantes aos da área com vegetação de cerrado (Tabela 4). Nas camadas mais profundas, os menores valores foram verificados na área de capim-tanzânia com 0,60 . Os valores de L, de IL e o IMC, à profundidade de $0-5 \mathrm{~cm}$, não diferiram entre si, em razão das coberturas e sistemas de manejo de solos estudados. Na profundidade de 5-10 cm, a L da matéria orgânica não diferiu entre as áreas de cultivo e foi menor, portanto, em relação à área de referência de vegetação

Tabela 3. Estoque de carbono orgânico total (COT), de carbono ligado à matéria orgânica particulada (C-MOP) e associado à fração mineral $(\mathrm{C}-\mathrm{MOM})$ do solo e suas percentagens em relação ao carbono total, com diferentes coberturas vegetais, manejado sob diferentes históricos de uso e manejo ${ }^{(1)}$.

\begin{tabular}{|c|c|c|c|c|c|}
\hline \multirow{2}{*}{$\begin{array}{l}\text { Cobertura } \\
\text { do solo }\end{array}$} & COT & C-MOP & C-MOM & C-MOP & C-MOM \\
\hline & \multicolumn{3}{|c|}{-------------(Mg ha'-1)-------------' } & \multicolumn{2}{|c|}{-----------(\%)-------- } \\
\hline & \multicolumn{5}{|c|}{$0-5 \mathrm{~cm}$} \\
\hline Milho & $14,79 b$ & $1,41 \mathrm{a}$ & $13,37 \mathrm{ab}$ & 9,5 & 90,5 \\
\hline Braquiária & $20,23 a$ & $3,01 \mathrm{a}$ & $16,42 \mathrm{a}$ & 14,9 & 85,1 \\
\hline Capim-tanzânia & $15,28 b$ & $3,28 \mathrm{a}$ & $12,30 \mathrm{~b}$ & 21,5 & 78,5 \\
\hline \multirow[t]{2}{*}{ Cerrado } & $15,00 \mathrm{~b}$ & $3,23 \mathrm{a}$ & $11,41 \mathrm{~b}$ & 21,5 & 78,5 \\
\hline & \multicolumn{5}{|c|}{$5-10 \mathrm{~cm}$} \\
\hline Milho & $15,04 \mathrm{a}$ & $0,66 \mathrm{cb}$ & $13,82 \mathrm{a}$ & 4,4 & 95,6 \\
\hline Braquiária & $16,68 \mathrm{a}$ & $1,55 b$ & $15,96 \mathrm{a}$ & 9,3 & 90,7 \\
\hline Capim-tanzânia & $8,88 \mathrm{~b}$ & $0,43 \mathrm{c}$ & $8,48 \mathrm{~b}$ & 4,8 & 95,2 \\
\hline \multirow[t]{2}{*}{ Cerrado } & $15,03 \mathrm{a}$ & $3,28 \mathrm{a}$ & $13,44 \mathrm{a}$ & 21,8 & 78,2 \\
\hline & \multicolumn{5}{|c|}{$10-20 \mathrm{~cm}$} \\
\hline Milho & $26,42 \mathrm{ab}$ & $1,05 \mathrm{~b}$ & $25,66 \mathrm{a}$ & 4,0 & 96,0 \\
\hline Braquiária & $23,29 \mathrm{ab}$ & $4,34 \mathrm{a}$ & $21,24 \mathrm{ab}$ & 18,6 & 81,4 \\
\hline Capim-tanzânia & $16,41 \mathrm{~b}$ & $0,68 b$ & $16,07 \mathrm{~b}$ & 4,1 & 95,9 \\
\hline Cerrado & $29,46 a$ & $4,33 \mathrm{a}$ & $25,44 \mathrm{a}$ & 14,7 & 85,3 \\
\hline$\overline{C V}(\%)$ & 17,92 & 39,91 & 14,04 & - & - \\
\hline
\end{tabular}

(1)Médias seguidas de letras iguais, nas colunas, não diferem entre si pelo teste de Dunn, a 5\% de probabilidade. 
de cerrado. Na profundidade de 10-20 cm, a área com braquiária apresentou maior labilidade. De forma semelhante, nessa mesma cobertura, ocorreram os maiores IL nas profundidades de 5-10 e 10-20 cm. Em todas as coberturas, a L da MOS apresentou decréscimo em profundidade, marcadamente na área com capim-tanzânia (Tabela 4).

O maior IMC do solo ocorreu na área de braquiária, com 44,01 e 111,61, nas profundidades de 5-10 e 10-20 cm respectivamente. No entanto, nas áreas de capim-tanzânia e de milho, principalmente nas camadas mais profundas, os valores de IMC foram baixos. Salton (2005) observou IMC superior para sistemas com presença de pastagem, com valores de 137 e 104, respectivamente, para área de pastagem permanente (U. decumbens) e rotação de soja/braquiária (soja por dois anos/U. decumbens), os quais superaram os sistemas apenas com lavoura e a vegetação de cerrado de referência. Os resultados observados no presente trabalho corroboram os de Salton (2005), na área de braquiária, o que sugere que o sistema de integração lavoura-pecuária contribuiu para o aumento na quantidade de MOS, com melhoria na qualidade do solo.

O sistema de manejo adotado, que integra a sucessão de lavouras de soja e milho com pecuária (animais e pastagens), tem contribuído para a manutenção

Tabela 4. Índices de estoque de carbono (IEC), labilidade (L), índice de labilidade (IL) e índice de manejo do carbono (IMC) do solo, com diferentes coberturas vegetais, manejado sob diferentes históricos de uso e manejo ${ }^{(1)}$.

\begin{tabular}{lcccr}
\hline Cobertura do solo & IEC & \multicolumn{4}{c}{ L } & IL & IMC \\
\hline Milho & \multicolumn{5}{c}{$0-5 \mathrm{~cm}$} \\
Braquiária & $1,99 \mathrm{~b}$ & $0,11 \mathrm{a}$ & $0,41 \mathrm{a}$ & $41,73 \mathrm{a}$ \\
Capim-tanzânia & $1,02 \mathrm{~b}$ & $0,19 \mathrm{a}$ & $0,74 \mathrm{a}$ & $101,77 \mathrm{a}$ \\
Cerrado & $1,00 \mathrm{~b}$ & $0,28 \mathrm{a}$ & $0,95 \mathrm{a}$ & $103,25 \mathrm{a}$ \\
& \multicolumn{5}{c}{$5-10 \mathrm{~cm}$} \\
Milho & $1,01 \mathrm{a}$ & $0,05 \mathrm{~b}$ & $0,20 \mathrm{c}$ & $100,00 \mathrm{a}$ \\
Braquiária & $1,11 \mathrm{a}$ & $0,09 \mathrm{~b}$ & $0,40 \mathrm{~b}$ & $21,17 \mathrm{c}$ \\
Capim-tanzânia & $0,60 \mathrm{~b}$ & $0,05 \mathrm{~b}$ & $0,21 \mathrm{c}$ & $12,74 \mathrm{c}$ \\
Cerrado & $1,00 \mathrm{a}$ & $0,24 \mathrm{a}$ & $1,00 \mathrm{a}$ & $100,00 \mathrm{a}$ \\
\hline & \multicolumn{5}{c}{$10-20 \mathrm{~cm}$} \\
Milho & $0,90 \mathrm{ab}$ & $0,04 \mathrm{~b}$ & $0,24 \mathrm{c}$ & $21,16 \mathrm{~b}$ \\
Braquiária & $0,80 \mathrm{ab}$ & $0,21 \mathrm{a}$ & $1,41 \mathrm{a}$ & $111,61 \mathrm{a}$ \\
Capim-tanzânia & $0,60 \mathrm{~b}$ & $0,04 \mathrm{~b}$ & $0,28 \mathrm{bc}$ & $16,28 \mathrm{~b}$ \\
Cerrado & $1,00 \mathrm{a}$ & $0,18 \mathrm{a}$ & $1,00 \mathrm{ab}$ & $100,00 \mathrm{a}$ \\
\hline CV (\%) & 14,83 & 42,70 & 42,50 & 51,17 \\
\hline
\end{tabular}

${ }^{(1)}$ Médias seguidas de letras iguais, nas colunas, não diferem entre si pelo teste de Dunn, a 5\% de probabilidade. dos atributos químicos favoráveis do solo e indica a adequação desse manejo, como também observado por Oliveira et al. (2002) e Perez et al. (2004).

Corazza et al. (1999) verificaram taxa anual de acúmulo de $1,43 \mathrm{Mg} \mathrm{ha}^{-1}$ de $\mathrm{C}$ em solo sob plantio direto no Cerrado. Porém, mesmo sob plantio direto, o C orgânico do solo é bastante dependente das culturas utilizadas em rotação. Bayer et al. (2004), em trabalho com diferentes coberturas na sucessão soja-milho sob plantio direto, verificaram incremento significativo de COT, em relação ao plantio convencional, apenas para a cobertura de nabo-forrageiro.

\section{Conclusões}

1. O manejo de áreas agrícolas que integre a sucessão de lavouras de grãos anuais com pastagens, contribui para a manutenção do estoque da matéria orgânica do solo e para a qualidade de seus atributos químicos.

2. O acúmulo de carbono orgânico no solo ocorre, preferencialmente, sob a forma de matéria orgânica particulada, que é a fração mais sensível a alterações no manejo do solo.

3. Em geral, a pastagem de braquiária é a cobertura de solo que proporciona índices de manejo de carbono no solo mais próximos aos da vegetação de cerrado.

\section{Agradecimentos}

À Fundação de Apoio ao Desenvolvimento do Ensino, Ciência e Tecnologia do Estado de Mato Grosso do Sul e ao Conselho Nacional de Desenvolvimento Científico e Tecnológico, pelo apoio financeiro.

\section{Referências}

ANGHINONI, I. Fertilidade do solo e seu manejo em sistema plantio direto. In: NOVAIS, R.F.; ALVAREZ V., V.H.; BARROS, N.F. de; FONTES, R.L.F.; CANTARUTTI, R.B.; NEVES, J.C.L. (Ed.). Fertilidade do solo. Viçosa: Sociedade Brasileira de Ciência do Solo, 2007. p.873-928.

BALBINOT JUNIOR, A.A.; MORAES, A. de; VEIGA, M. da; PELISSARI, A.; DIECKOW J. Integração lavoura-pecuária: intensificação de usos de áreas agrícolas. Ciência Rural, v.39, p.1925-1933, 2009.

BAYER, C. Dinâmica da matéria orgânica em sistemas de manejo de solos. 1996. 241p. Tese (Doutorado)- Universidade Federal do Rio Grande do Sul, Porto Alegre.

BAYER, C.; MARTIN-NETO, L.; MIELNICZUK, J.; PAVINATO, A. Armazenamento de carbono em frações lábeis da matéria 
orgânica de um Latossolo Vermelho sob plantio direto. Pesquisa Agropecuária Brasileira, v.39, p.677-683, 2004.

BAYER, C.; MARTIN-NETO, L.; MIELNICZUK, J.; PILLON, C.N.; SANGOI, L. Changes in soil organic matter fractions under subtropical no-till cropping systems. Soil Science Society of America Journal, v.65, p.1473-1478, 2001.

BAYER, C.; MIELNICZUK, J.; MARTIN-NETO, L.; ERNANI, P.R. Stocks and humification degree of organic matter fractions as affected by no-tillage on a subtropical soil. Plant and Soil, v.238, p.133-140, 2002.

BLAIR, G.J.; LEFROY, R.D.B.; LISLE, L. Soil carbon fractions based on their degree of oxidation, and the development of a carbon management index for agricultural systems. Australian Journal of Agricultural Research, v.46, p.1459-1466, 1995.

BODDEY, R.M.; JANTALIA, C.P.; ALVES, B.J.R.; URQUIAGA, S. Comments on "no-tillage and soil-profile carbon sequestration: an on-farm assessment". Soil Science Society of America Journal, v.73, p.688-689, 2008.

CAMBARDELLA, C.A.; ELLIOTT, E.T. Particulate soil organic matter change across a grassland cultivation sequence. Soil Science Society of America Journal, v.56, p.777-783, 1992.

CHAN, K.Y. Consequences of changes in particulate organic carbon in Vertisols under pasture and cropping. Soil Science Society of America Journal, v.61, p.1376-1382, 1997.

CHRISTENSEN, B.T. Physical fractionation of soil and structural and functional complexity in organic matter turnover. European Journal of Soil Science, v.52, p.345-353, 2001.

CLAESSEN, M.E.C. (Org.). Manual de métodos de análise de solo. 2.ed. rev. atual. Rio de Janeiro: Embrapa-CNPS, 1997. 212p. (Embrapa-CNPS. Documentos, 1).

CONCEIÇÃO, P.C.; AMADO, T.J.C.; MIELNICZUK, J.; SPAGNOLLO, E. Qualidade do solo em sistemas de manejo avaliada pela dinâmica da matéria orgânica e atributos relacionados. Revista Brasileira de Ciência do Solo, v.29, p.777-788, 2005.

CORAZZA, E.J.; SILVA, J.E.; RESCK, D.V.S.; GOMES, A.C. Comportamento de diferentes sistemas de manejo como fonte ou depósito de carbono em relação à vegetação de cerrado. Revista Brasileira de Ciência do Solo, v.23, p.425-432, 1999.

COSTA, F.S.; ALBUQUERQUE, J.A.; BAYER, C.; FONTOURA, S.M.V.; WOBETO, C. Propriedades físicas de um Latossolo Bruno afetadas pelos sistemas plantio direto e preparo convencional. Revista Brasileira de Ciência Solo, v.27, p.527-535, 2003.

FELLER, C.; BEARE, M.H. Physical control of soil organic matter dynamics in the tropics. Geoderma, v.79, p.69-116, 1997.

FRANZLUEBBERS, A.J.; ARSHAD, M.A. Particulate organic carbon content and potential mineralization as affected by tillage and texture. Soil Science Society of America Journal, v.61, p.1382-1386, 1997.

FREIXO, A.A.; MACHADO, P.L.O.A.; GUIMARÃES, C.M.; SILVA, C.A.; FADIGAS, F.S. Estoque de carbono e nitrogênio e distribuição de frações orgânicas de Latossolo do Cerrado sob diferentes sistemas de cultivo. Revista Brasileira de Ciência do Solo, v.26, p.425-434, 2002.

GOLCHIN, A.; OADES, J.M.; SKJEMSTAD, J.O.; CLARKE, P. Study of free and occluded particulate organic matter in soils by solid state $13 \mathrm{C} \mathrm{CP/MAS} \mathrm{NMR} \mathrm{spectroscopy} \mathrm{and} \mathrm{scanning}$ electron microscopy. Australian Journal of Soil Research, v.32, p.285-309, 1994.

NEEDELMAN, B.A.; WANDER, M.M.; BOLLERO, G.A.; BOAST, C.W.; SIMS, G.K.; BULLOCK, D.G. Interaction of tillage and soil texture: biologically active soil organic matter in Illinois. Soil Science Society of America Journal, v.63, p.1326-1334, 1999.

NICOLOSO, R. da S.; LOVATO, T.; AMADO, T.J.C.; BAYER, C.; LANZANOVA, M.E. Balanço do carbono orgânico no solo sob integração lavoura-pecuária no Sul do Brasil. Revista Brasileira de Ciência do Solo, v.32, p.2425-2433, 2008.

OLIVEIRA, F.C.; MATTIAZZO, M.E.; MARCIANO, C.R.; ABREU JÚNIOR, C.H. Alterações em atributos químicos de um Latossolo pela aplicação de composto de lixo urbano. Pesquisa Agropecuária Brasileira, v.37, p.529-538, 2002.

PEREZ, K.S.S.; RAMOS, M.L.G.; MCMANUS, C. Carbono da biomassa microbiana em solo cultivado com soja sob diferentes sistemas de manejo nos Cerrados. Pesquisa Agropecuária Brasileira, v.39, p.567-573, 2004.

SALTON, J.C. Matéria orgânica e agregação do solo na rotação lavoura-pastagem em ambiente tropical. 2005. 157p. Tese (Doutorado) - Universidade Federal do Rio Grande do Sul, Porto Alegre.

SANTOS, H.G. dos; JACOMINE, P.K.T.; ANJOS, L.H.C. dos; OLIVEIRA, V.A. de; OLIVEIRA, J.B. de; COELHO, M.R.; LUMBRERAS, J.F.; CUNHA, T.J.F. (Ed.). Sistema brasileiro de classificação de solos. 2.ed. Rio de Janeiro: Embrapa Solos, 2006. 306p.

SCHLINDWEIN, J.A.; GIANELLO, C. Necessidade de novos estudos de calibração e recomendação de fertilizantes para as culturas cultivadas sob sistema plantio direto. Revista Plantio Direto, v.79, p.12-15, 2004.

SIX, J.; ELLIOTT, E.T.; PAUSTIAN, K.; DORAN, J.W. Aggregation and soil organic matter accumulation in cultivated and native grassland soils. Soil Science Society of America Journal, v.62, p.1367-1377, 1998.

SPERA, S.T.; SANTOS, H.P. dos; FONTANELI, R.S.; TOMM, G.O. Efeito de pastagens de inverno e de verão em características físicas de solo sob plantio direto. Ciência Rural, v.36, p.1193-1200, 2006.

TRACY, B.F.; ZHANG, Y. Soil compaction, corn yield response and soil nutrient pool dynamics within an integrated crop-livestock system in Illinois. Crop Science, v.48, p.1211-1218, 2008.

WANDER, M.M.; BIDART, M.G. Tillage impacts in depth distribution of total and particulate organics matter in three Illinois soils. Soil Science Society of America Journal, v.62, p.1704-1711, 1998.

Recebido em 12 de maio de 2010 e aprovado em 9 de agosto de 2011 